\title{
Review of guidelines related to the regulations on telehealth and internet- based prescribing from selected countries.
}

\author{
G.G.A.K. Kulatunga ${ }^{1}$, R. Hewapathirana ${ }^{2}$, R.B. Marasinghe ${ }^{3}$, V.H.W. Dissanayake ${ }^{2}$ \\ 1 Post Graduate Institute of Medicine, Colombo, Sri Lanka \\ 2 Faculty of Medicine, University of Colombo, Sri Lanka \\ 3 Department of Medical Education, Faculty of Medical Sciences, University of Sri Jayawardenapura, Sri Lanka \\ *gumindu@gmail.com \\ https://orcid.org/0000-0003-2164-5361
}

\begin{abstract}
Introduction: Telehealth related services are spreading rapidly throughout many countries of the world. Proper guidance on the provision of these services is essential to provide quality healthcare. Internet-based prescribing is an important component of telehealth services. If allowed, Internet-based prescribing must be done within a proper regulatory framework.

Method: Guidelines related to the regulation of telehealth services, from registration of physicians to practice medicine to delivery of medicine to patient through internet-based prescriptions were identified through a web-based search using standard medical/ health databases. These documents were reviewed and comparatively analysed.

Results: 14 regulatory guidelines were identified. They were from countries/ states with established digital health ecosystems such as Australia, Canada (country wide and for the states of British Columbia and Ontario), Denmark, France, New Zealand, Portugal, Singapore, United Kingdom, United states of America (for the states of California, Maine, North Carolina and Washington). The guidelines were related to the practice of Telemedicine including internet-based prescribing. These guidelines were uniform in relation to the following: any medical prescription was valid, if it is prescribed/issued by a health professional with a licence to prescribe; there was no distinction between 'telemedicine license' and the standard 'medical license'; in cross border practice, the physicians were required to have a licence to practice medicine in the country where the patient was a resident, at the time of care; general requirements needed to start telehealth services [ensuring that telehealth was appropriate to the situation; ensuring that there was a pre-established relationship with the patient in case of prescription of controlled medications; ensuring that the physician was able to access sufficient patient information prior to prescribing; training of physicians on providing the telehealth service]; ensuring that valid informed consent can be obtained; ensuring the privacy and confidentiality of patient data; ensuring that the patient knows the physician's identity, qualifications, and where he is providing the service from.; ensuring that the patients know the limitations of telehealth methodology; ensuring that patient identify can be confirmed prior to the consultation; ensuring that suitable distance diagnostic devises are used, and ensuring that medical records are retained.
\end{abstract}

Conclusion: The above-mentioned guidelines would be useful in developing a telehealth regulatory framework for Sri Lanka.

Keywords: Guidelines, Regulations, Telehealth, Internet-based prescribing 